\title{
PROPOSIÇÃO DE UM INSTRUMENTO DE MENSURAÇÃO DO DESENVOLVIMENTO DE CAPACIDADES DINÂMICAS
}

\author{
(iD) Felipe Cavalheiro Zaluski ${ }^{1}$ (D) Jorge Oneide Sausen², (iD) Gloria Charão Ferreira ${ }^{3}$ \\ ${ }^{1}$ Universidade Federal de Santa Maria - UFSM. Santa Maria, Rio Grande do Sul-Brasil.felipezaluski@ hotmail.com \\ ${ }^{2}$ Universidade Regional do Noroeste do Estado do Rio Grande do Sul - UNIJUÍ. Ijuí. Rio Grande do Sul - Brasil. josausen@unijui.edu.br \\ ${ }^{3}$ Universidade Regional do Noroeste do Estado do Rio Grande do Sul - UNIJUÍ. Ijuí. Rio Grande do Sul - Brasil. gcfconsultoria@ gmail.com
}

\begin{abstract}
Resumo
Objetivo: este estudo objetiva construir e validar uma escala de mensuração dos elementos componentes e dos mecanismos organizacionais de desenvolvimento de capacidades dinâmicas (CD) em organizações. Método: a metodologia da pesquisa baseou-se na abordagem quantitativa e descritiva, com coleta de dados bibliográfica e por meio de e-survey em 137 organizações do Sul do Brasil. A validação da escala foi realizada pela modelagem de equações estruturais (MEE) com o software SmartPLS 3.2.8. Originalidade/Relevância: ainda que existam esforços no desenvolvimento de escalas sobre $\mathrm{CD}$, há uma lacuna teórica quanto à criação de escalas e instrumentos de medição, uma vez que tais capacidades estão ligadas aos processos internos das organizações. Diante disso, este estudo se mostra relevante ao se direcionar à proposição e validação de uma escala baseada no desenvolvimento das CD que possibilite novas investigações empíricas e discussões teóricas.

Resultados: a escala final proposta para mensuração dos elementos componentes e dos mecanismos organizacionais de desenvolvimento de $\mathrm{CD}$ apresentou três dimensões e vinte indicadores. Os achados demonstram que a articulação dos indicadores dos elementos componentes e dos mecanismos de desenvolvimento das capacidades dinâmicas resulta em uma escala que pondera vários aspectos relacionados à existência do desenvolvimento de $\mathrm{CD}$ em organizações.

Contribuições teóricas/metodológicas: esta pesquisa proporciona um recurso acadêmico para a utilização da escala proposta em futuras pesquisas e amplia a possibilidade de desenvolvimento da temática das capacidades dinâmicas nos estudos organizacionais.
\end{abstract}

Palavras-chave: Escala. Processos. Rotinas.

\section{PROPOSITION AND VALIDATION OF A MEASUREMENT SCALE FOR DYNAMIC CAPACITY DEVELOPMENT IN ORGANIZATIONS}

\section{Abstract}

Objective: this study aims to build and validate a measurement scale of the component elements and the organizational mechanisms for the development of dynamic capabilities (DC) in organizations.

Method: the research methodology was based on a quantitative and descriptive approach, with bibliographic data collection and through an esurvey in 137 organizations in southern Brazil. The scale validation was performed by structural equation modeling (SEM) with the SmartPLS 3.2.8 software.

Originality/Relevance: although there are efforts in the development of scales on DC, there is a theoretical gap regarding the creation of scales and measurement instruments, since these capacities are linked to the internal processes of organizations. In view of this, this study proves to be relevant in addressing the proposition and validation of a scale based on the development of DC that allows new empirical investigations and theoretical discussions.

Results: the final scale proposed for measuring the component elements and the organizational mechanisms for $\mathrm{CD}$ development had three dimensions and twenty indicators. The findings demonstrate that the articulation of the indicators of the component elements and the mechanisms for the development of dynamic capacities results in a scale that weighs several aspects related to the existence of DC development in organizations.

Theoretical / methodological contributions: this research provides an academic resource for the use of the scale proposed in future research and expands the possibility of developing the theme of dynamic capabilities in organizational studies.

Keywords: Scale. Process. Routines.

\section{PROPUESTA DE UN INSTRUMENTO PARA MEDIR EL DESARROLLO DE CAPACIDADES DINÁMICAS}

\section{Resumen}

Objetivo: este estudio tiene como objetivo construir y validar una escala de medición de los elementos componentes y los mecanismos organizacionales para el desarrollo de capacidades dinámicas (CD) en las organizaciones.

Método: la metodología de investigación se basó en un enfoque cuantitativo y descriptivo, con recolección de datos bibliográficos y mediante encuesta electrónica en 137 organizaciones del sur de Brasil. La validación de la escala se realizó mediante el modelado de ecuaciones estructurales (SEM) con el software SmartPLS 3.2.8

Originalidad/Relevancia: si bien existen esfuerzos en el desarrollo de escalas en $\mathrm{CD}$, existe un vacío teórico en cuanto a la creación de escalas e instrumentos de medición, ya que dichas capacidades están ligadas a los procesos internos de las organizaciones. Ante esto, este estudio resulta relevante para abordar la proposición y validación de una escala basada en el desarrollo de las CD que posibilite nuevas investigaciones empíricas y discusiones teóricas.

Resultados: la escala final propuesta para medir los elementos componentes y los mecanismos organizativos para el desarrollo del DC tuvo tres dimensiones y veinte indicadores. Los hallazgos demuestran que la articulación de los indicadores de los elementos componentes y los mecanismos para el desarrollo de capacidades dinámicas resulta en una escala que pondera varios aspectos relacionados con la existencia de desarrollo de $\mathrm{CD}$ en las organizaciones. Contribuciones teóricas/metodológicas: esta investigación proporciona un recurso académico para el uso de la escala propuesta en futuras investigaciones y amplía la posibilidad de desarrollar el tema de las capacidades dinámicas en los estudios organizacionales.

Palabras clave: Escala. Procesos. Rutinas. 


\section{Introdução}

Buscando explicar como as organizações agem para reconfigurar de forma proativa sua base de recursos, a perspectiva teórica das capacidades dinâmicas $(\mathrm{CD})$ pondera a habilidade de a organização integrar, construir e reconfigurar as competências externas e internas para adaptar-se rapidamente ao ambiente altamente mutável (Sausen, 2012; Teece, Pisano, \& Shuen, 1997). Nesse contexto, a teoria das CD surge como uma nova abordagem da administração estratégica que, ao combinar o dinamismo do ambiente e os fatores internos da organização, é capaz de gerar vantagem competitiva sustentável (Teece, 2007; Teece et al., 1997; Wang \& Ahmed, 2007; Zahra \& George, 2002).

As CD utilizam os pressupostos teóricos da visão baseada em recursos (resource-based view, ou RBV) para explicar e compreender como as organizações buscam reformular seus recursos organizacionais a fim de adaptar-se às mudanças ocorridas no ambiente (Teece et al., 1997). Diversos autores (Ambrosini \& Bowman, 2009; Eisenhardt \& Martin, 2000; Ferreira, Reis, Serra, \& Costa, 2014; Helfat, 1997; Marcus \& Anderson, 2006; Teece, 2007; Teece \& Pisano, 1994; Teece et al., 1997) defendem que a teoria das $\mathrm{CD}$ surge para preencher a lacuna teórica deixada pela RBV, no sentido de tornar a vantagem competitiva sustentável em ambientes altamente dinâmicos e explicar como algumas organizações respondem rapidamente às mudanças do ambiente externo.

Os primeiros estudos sobre as CD foram iniciados por Winter (1964). Contudo, esse tema passou a ter maior relevância a partir da década de 90, com os estudos de Teece e Pisano (1994), Teece et al. (1997) e Eisenhardt e Martin (2000), o que levou a um aumento das pesquisas no âmbito estratégico em virtude da redução das fronteiras entre os mercados e da consequente elevação da concorrência entre as organizações (Martins \& Tavares, 2014). Desde a sua primeira abordagem, essa temática tem ganhado destaque em investigações na área da Administração, atraindo atenção como um campo de estudo promissor (Barreto, 2010; Sher \& Lee, 2004) em pesquisas internacionais (Ambrosini \& Bowman, 2009; Barreto, 2010; Easterby-Smith \& Pietro, 2008) e nacionais (Guerra, Tondolo, \& Camargo, 2016).

Diante disso, Barreto (2010) e Eriksson (2014) apontam a necessidade de constructos multidimensionais para avaliação das CD. Nesse contexto, são necessários estudos quantitativos que possam proporcionar instrumentos para elucidar o modo como as organizações desenvolvem CD. Algumas pesquisas já objetivaram propor e validar constructos, escalas ou instrumentos de mensuração e/ou avaliação do desenvolvimento de capacidades turísticas (Silveira-Martins \& Zonatto, 2015), capacidades dinâmicas produtivas (D'avila \& Silveira-Martins, 2017), capacidades dinâmicas do agronegócio (Silveira \& Silveira-Martins, 2017), capacidades dinâmicas do terceiro setor (Tondolo, Tondolo, Guerra, \& Camargo, 2017), capacidades dinâmicas para serviços ágeis (Raman \& Bharadwaj, 2017), capacidades de percepção, apreensão e transformação (Kump, Engelmann, Kessler, \& Schweiger, 2018) e capacidades gerenciais dinâmicas (Corrêa, Bueno, Karto, \& Silva, 2018).

Araújo, Pedron e Bitencourt (2018) evidenciam que o número reduzido de desenvolvimento de escalas sobre CD advém da dificuldade em propor medições dessas capacidades, uma vez que elas estão 
ligadas aos processos internos das organizações (Teece, 2007). Ainda que existam esforços para o desenvolvimento de escalas sobre $\mathrm{CD}$, há uma lacuna teórica quanto à criação de escalas e instrumentos de medição confiáveis e válidos para as CD (Araújo et al., 2018). Tendo isso em vista, este estudo se mostra relevante ao se direcionar à proposição e validação de uma escala baseada no desenvolvimento das CD que possibilite tanto novos estudos quanto comparações ou futuras metanálises da temática (Kump et al., 2018).

Assim, esta pesquisa objetiva propor e validar uma escala de mensuração dos elementos componentes e dos mecanismos organizacionais de desenvolvimento de CD em organizações. Para a proposição das dimensões da escala, levou-se em consideração o modelo dos elementos componentes e dos mecanismos organizacionais de desenvolvimento das CD de Meirelles e Camargo (2014), que aborda: i) comportamentos e habilidades de mudança e inovação; ii) processos e rotinas de busca e inovação; e iii) mecanismos de aprendizagem e governança do conhecimento. E, para a proposição dos indicadores de cada dimensão, analisaram-se estudos empíricos que adotaram o modelo teórico supracitado (Cappellari, 2017; Lazarotto, 2017; Rosa, 2016; Schmitz, 2018; Silva, 2016; Welter, 2018).

Para a consecução da pesquisa, adotou-se uma metodologia quantitativa e descritiva, com coleta de dados por meio de $e$-survey em uma amostra de 137 organizações localizadas na região Noroeste do Rio Grande do Sul. O desenvolvimento do estudo iniciou com a proposição das dimensões e dos indicadores da escala e posterior validação do modelo de mensuração, passando, por fim, à validação do modelo estrutural da escala e às discussões teóricas dos resultados. As conclusões demonstram que a escala final proposta para mensuração dos elementos componentes e dos mecanismos organizacionais de desenvolvimento de $\mathrm{CD}$ apresentou três dimensões e vinte indicadores. Os resultados apontam que a articulação dos indicadores dos elementos componentes e dos mecanismos de desenvolvimento das CD resulta em uma escala que pondera vários aspectos relacionados à existência do desenvolvimento de CD em organizações.

\section{Capacidades dinâmicas}

A teoria das CD constitui-se como um campo de estudos de grande interesse para pesquisadores na área de Administração (Meirelles \& Camargo, 2014). O conceito de CD é relevante porque trata da capacidade da organização ante o dinamismo do ambiente, surgindo pela importância de analisar a forma pela qual as organizações alcançam e sustentam vantagens competitivas em um ambiente dinâmico (Teece, 2009; Teece et al., 1997; Vasconcelos \& Cyrino, 2000). Neste estudo, adota-se a definição de CD de Teece (2007, p. 1319): "podem ser desagregadas em capacidade (a) de sentir e dar forma às oportunidades e ameaças; (b) para aproveitar as oportunidades; (c) para manter a competitividade através do reforço, da combinação, protegendo e, quando necessário, reconfigurando os bens corpóreos e incorpóreos da empresa". 
Essa teoria, que é concebida como uma extensão da RBV de Penrose (1959), analisa a organização a partir de um conjunto de recursos, tendo sido amplamente disseminada por Barney (1991). Desse modo, diferentemente da abordagem da RBV, que é mais estática e aborda a vantagem competitiva por meio dos recursos internos da organização, a perspectiva das CD compreende a vantagem competitiva em ambientes dinâmicos, que estão em constante mudança (Ambrosini \& Bowman, 2009; Eisenhardt \& Martin, 2000; Kurtmollaiev, 2020; Schilke, Hu, \& Helfat, 2018; Teece et al., 1997).

Vários autores vêm buscando desenvolver o conceito de CD, sobretudo do ponto de vista dos seus microfundamentos e de sua operacionalização (Teece, 2009; Wang \& Ahmed, 2007; Zollo \& Winter, 2002). Na década de 90, Teece, Pisado e Shuen (1990) iniciaram essa empreitada teórica, destacando que não apenas o conjunto de recursos que mantém a vantagem competitiva é importante, como apregoa a RBV, mas, também, os mecanismos pelos quais as organizações desenvolvem novas habilidades e capacidades.

Teece e Pisano (1994) conceituam a formação da expressão "capacidade dinâmica", explicitando que o termo "dinâmico" remete à capacidade de renovar as competências para se alinhar com o ambiente competitivo de rápidas mudanças, de alto grau de inovação, de novas tecnologias e de alta concorrência do mercado, cuja natureza é difícil determinar. Já o termo "capacidades", conforme os autores, enfatiza o papel fundamental da administração estratégica em buscar adaptar, integrar e reconfigurar as competências, os recursos funcionais e as habilidades organizacionais internas e externas diante das mudanças do ambiente considerado (Teece \& Pisano, 1994).

Além das várias definições encontradas na literatura, diversas são as designações quanto aos seus constructos (ou elementos componentes dessas capacidades em uma organização). Segundo Meirelles e Camargo (2014), enquanto parte dos autores enfatizam as CD como um conjunto de processos e rotinas organizacionais, caso de Eisenhardt e Martin (2000), Teece et al. (1997), Zahra, Sapienza e Davidsson (2006) e Zollo e Winter (2002), outra parte ressalta habilidades, comportamentos e capacidades organizacionais, como fazem Andreeva e Chaika (2006), Collis (1994), Helfat, Finkelstein, Mitchell, Peteraf, Singh, Teece, \& Winter (2007) e Wang e Ahmed (2007).

Com relação ao primeiro grupo, pode-se citar os entendimentos de Teece et al. (1997), para quem as CD são operacionalizadas na forma de fatores compostos de processos, posições e trajetórias, assim como de Eisenhardt e Martin (2000), que mencionam como componentes das CD os processos ou as rotinas organizacionais e as estratégias pelas quais a organização alcança novas configurações de recursos, e de Zollo e Winter (2002), que indicam os processos de acumulação de experiências, de articulação e de codificação do conhecimento e, ainda, o ciclo recursivo de aprendizagem. Também integrando esse primeiro grupo, Zahra et al. (2006) referem a existência de uma hierarquia de capacidades, propondo duas categorias: (i) capacidades substantivas ou recursos utilizados para resolver problemas do cotidiano; e (ii) capacidades dinâmicas relacionadas à habilidade da empresa em 
transformar ou reconfigurar as suas capacidades substantivas ou comuns em novas capacidades dinâmicas.

Entre os autores que enfatizam as CD como um conjunto de capacidades, habilidades e comportamentos, está Collis (1994), que autor menciona as capacidades operacionais, as habilidades em desenvolver novas estratégias rapidamente (ou melhor do que a concorrência) e a capacidade de aprender a aprender como elementos componentes das $\mathrm{CD}$, destacando, portanto, a velocidade de operação perante a concorrência. Da mesma forma, Helfat et al. (2007) sugerem a habilidade para alterar a base de recursos, a capacidade de atuar de modo recorrente para modificar essa base e a capacidade de buscar e selecionar aquelas modificações relevantes como sendo os elementos componentes das CD, ressaltando, assim, o poder de deliberação da empresa.

Conforme afirmam Guerra et al. (2016) - baseados nos estudos de Ambrosini e Bowman (2009), Danneels (2010), Helfat et al. (2007) e Zahra et al. (2006) -, as CD podem ser fonte de competitividade se os processos de mudança da base de recursos e de capacidades organizacionais forem intencionais, ou seja, dirigidos pela gestão das organizações face às exigências competitivas do mercado. Para Zahra et al. (2006), as empresas criam, definem, descobrem e exploram as oportunidades, frequentemente antes dos seus rivais, criando valor por meio das suas $\mathrm{CD}$ e reconfigurando os recursos e as rotinas de acordo com a percepção da organização ou do empreendedor.

Nesse sentido, as rotinas e os mecanismos organizacionais podem ser observados como base de estudo e entendimento das CD (Eisenhardt \& Martin, 2000; Zahra et al., 2006). Em relação a rotinas, Winter (2003) diz que uma capacidade organizacional é uma rotina de alto nível ou um conjunto de rotinas que atribuam à gestão organizacional um conjunto de opções capazes de produzir resultados significativos e diferenciados para a organização. Imprime-se aqui um significado diferente à palavra "rotina", na medida em que estudos evidenciam a existência de rotinas e práticas que irão alavancar projetos e desenvolver capacidades dinâmicas (Zahra et al., 2006).

Elementos componentes e mecanismos organizacionais de desenvolvimento das capacidades dinâmicas

Meirelles e Camargo (2014) propõem uma síntese dos elementos componentes e dos mecanismos de desenvolvimento das $\mathrm{CD}$, a partir da realização de uma pesquisa bibliográfica acerca da temática. Nesse contexto, os autores destacam que é necessário que a empresa desenvolva comportamentos e habilidades de mudança e inovação, processos e rotinas de busca e inovação e mecanismos de aprendizagem e governança do conhecimento que deem suporte à existência de $\mathrm{CD}$. $\mathrm{Ou}$ seja, deve haver um ciclo contínuo de acumulação de experiência por meio de um processo recursivo que torne a empresa cada vez mais apta a desenvolver tal capacidade.

A Figura 1, a seguir, demonstra comportamentos e habilidades de mudança e inovação, associados aos processos e às rotinas de suporte, os quais são sustentados por mecanismos de aprendizagem e governança do conhecimento, resultando em CD. 
Figura 1 - Modelo integrado de capacidades dinâmicas

\section{EIementos componentes}

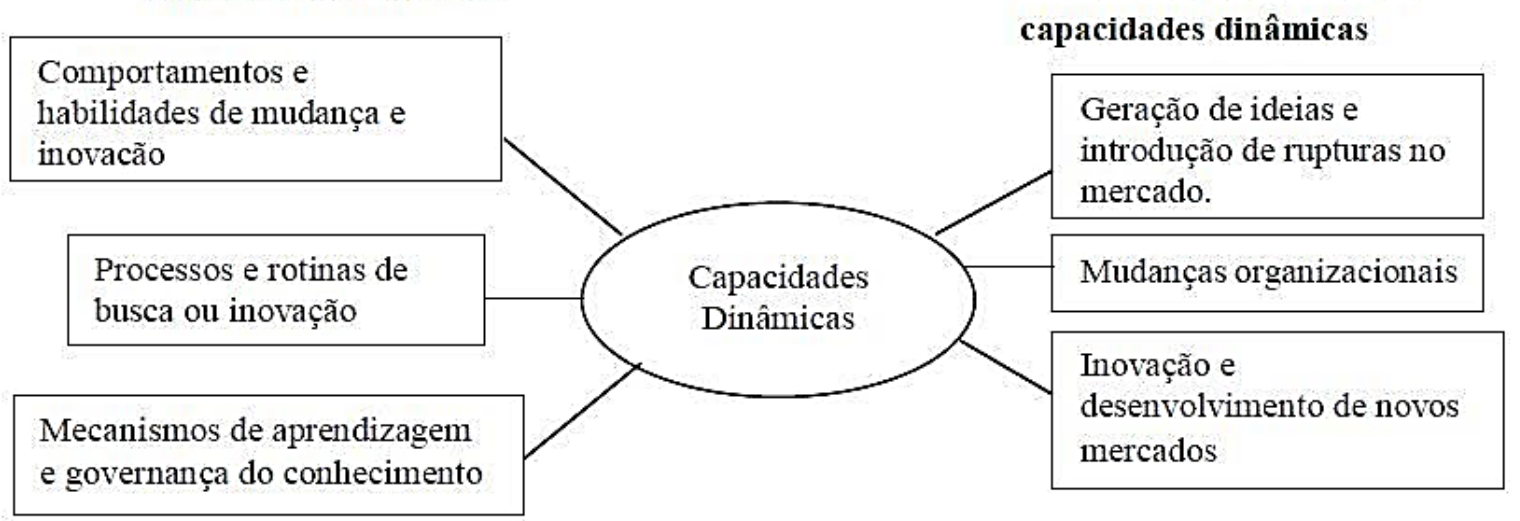

Fonte: Camargo e Meirelles (2012, p. 14).

Portanto, na visão de Meirelles e Camargo (2014), para que as CD se desenvolvam nas organizações, são necessários três elementos componentes: comportamentos e habilidades; processos e rotinas; e mecanismos de aprendizagem e governança do conhecimento. As $\mathrm{CD}$, por resultarem desse conjunto de elementos e mecanismos de suporte, podem ser identificadas e mensuradas a partir de indicadores relacionados à geração de ideias, à introdução de rupturas no mercado, às mudanças organizacionais, à inovação e ao desenvolvimento de novos mercados (Meirelles \& Camargo, 2014). Destaca-se, assim, uma evolução nos estudos no sentido de relacionar trabalhos de importantes autores e elaborar uma proposta considerando esses fatores como elementos componentes e mecanismos de desenvolvimento de CD.

De acordo com Meirelles e Camargo (2014), para que uma organização desenvolva CD, é preciso um conjunto de comportamentos e habilidades de mudança e inovação, como também processos e rotinas que deem suporte à inovação, além de certos mecanismos de aprendizagem que sustentem formas de governança do conhecimento. Dessa forma, o comportamento está relacionado à maneira como os indivíduos comportam-se, reagem e portam-se perante estímulos advindos do seu entorno.

Quanto às habilidades, Andreeva e Chaika (2006) sugerem que, para uma organização criar capacidade de mudança, entre outros fatores, é importante o desenvolvimento de habilidades não específicas. Essas habilidades têm um âmbito de aplicação mais vasto, pois não se limitam apenas às atividades rotineiras dos colaboradores, permitindo que eles se sintam menos limitados e tenham capacidade de agir em prol de qualquer tipo de mudança.

Além das habilidades e dos comportamentos, Teece (2009) destaca a necessidade de associação desses elementos a rotinas e processos de suporte para a geração de novas ideias, produtos e serviços, bem como para a seleção e implementação da mudança. Gerard (2011) explana que os processos contêm todas as capacidades necessárias para transformar entradas em resultados desejados, englobando toda a estrutura de trabalho existente na organização. Nesse sentido, a organização, como mencionam Zollo e 
Winter (2002), pode ser entendida como um conjunto de rotinas operacionais e administrativas que evoluem ao longo do tempo por meio do feedback do desempenho.

Juntamente às rotinas e aos processos, empresa deve desenvolver mecanismos de aprendizagem e governança do conhecimento que forneçam suporte à existência de CD (Meirelles \& Camargo, 2014). Para Zollo e Winter (2002), as CD são desenvolvidas por intermédio da coevolução de mecanismos de aprendizagem, visto que esses constituem métodos sistemáticos para modificar as rotinas operacionais. Portanto, neste estudo, considera-se o tripé defendido por Meirelles e Camargo (2014) - comportamento e habilidades; processos e rotinas; e mecanismos de aprendizagem e governança do conhecimento para a proposição da escala de mensuração dos elementos componentes das $\mathrm{CD}$, que é discutida na sequência.

\section{Definição das dimensões e dos indicadores da escala}

O desenvolvimento das $\mathrm{CD}$ teve como âncora as três dimensões baseadas nos elementos componentes e nos mecanismos organizacionais propostos por Meirelles e Camargo (2014): i) comportamentos e habilidades de mudança e inovação; ii) processos e rotinas de busca e inovação; e iii) mecanismos de aprendizagem e governança do conhecimento. Os indicadores de desenvolvimento das CD foram elucidados com base nos estudos de Cappellari (2017), Lazarotto (2017), Rosa (2016), Schmitz (2018), Silva (2016) e Welter (2018). Esses estudos consideraram o desenvolvimento das CD fundamentados nos elementos componentes e mecanismos organizacionais propostos por Meirelles e Camargo (2014).

Primeiramente, analisaram-se os estudos selecionados destacando as evidências dos elementos componentes e dos mecanismos organizacionais de desenvolvimento das $\mathrm{CD}$ resultantes desses estudos. Após, consideraram-se as evidências semelhantes, sendo definida uma proposta de um indicadorsíntese. Posteriormente, efetuou-se a classificação dos indicadores propostos nas dimensões de análise, seguindo o modelo de Meirelles e Camargo (2014), e elaborou-se uma questão em forma de afirmativa para compor a proposição da escala.

A fim de validar o conteúdo da escala proposta, selecionaram-se três especialistas (com titulação mínima de doutorado e com experiência na temática desta pesquisa) para avaliar as dimensões, os indicadores e as afirmativas da escala. Após, efetuou-se um pré-teste com cinco empresas aleatórias da amostra adotada no estudo, que buscou ponderar acerca do preenchimento, da leitura e da compreensão das questões, assim como da estrutura e da apresentação da escala, conforme apresentado na Tabela 1. 
Tabela 1 - Proposição da escala de mensuração

\begin{tabular}{|c|c|c|c|c|c|}
\hline CÓD. & DIMENSÃO & CÓD. & INDICADOR & AFIRMATIVA & $\begin{array}{l}\text { EVIDÊNCIA } \\
\text { TEÓRICA }\end{array}$ \\
\hline \multirow{8}{*}{ CHMI } & \multirow{8}{*}{$\begin{array}{c}\text { COMPORTAMENTOS } \\
\text { E HABILIDADES DE } \\
\text { MUDANÇA E } \\
\text { INOVAÇÃO }\end{array}$} & CHMI1 & $\begin{array}{l}\text { Comprometimento } \\
\text { com a mudança }\end{array}$ & $\begin{array}{l}\text { A organização é } \\
\text { comprometida com a } \\
\text { mudança }\end{array}$ & $\begin{array}{l}\text { Cappellari (2017) } \\
\text { Lazarotto (2017) } \\
\text { Rosa (2016) } \\
\text { Silva (2016) } \\
\end{array}$ \\
\hline & & CHMI2 & $\begin{array}{c}\text { Interação } \\
\text { interdepartamental }\end{array}$ & \begin{tabular}{ll}
\multicolumn{2}{l}{ Existe diálogo e troca de } \\
experiência entre os \\
departamentos \\
$\begin{array}{ll}\text { organização } \\
\end{array}$ \\
\end{tabular} & $\begin{array}{l}\text { Schmitz (2018) } \\
\text { Rosa (2016) } \\
\text { Lazarotto (2017) }\end{array}$ \\
\hline & & CHMI3 & $\begin{array}{l}\text { Habilidade de } \\
\text { resolubilidade }\end{array}$ & $\begin{array}{l}\text { A organização possui } \\
\text { metodologia } \\
\text { habilidade para resolver } \\
\text { problemas }\end{array}$ & $\begin{array}{l}\text { Cappellari (2017) } \\
\text { Lazarotto (2017) } \\
\text { Rosa (2016) } \\
\text { Welter (2018) } \\
\end{array}$ \\
\hline & & CHMI4 & $\begin{array}{l}\text { Pioneirismo } \\
\text { tecnológico }\end{array}$ & $\begin{array}{ll}\text { A organização } & \text { busca } \\
\text { constantemente } & \text { novas } \\
\text { tecnologias } & \\
\end{array}$ & $\begin{array}{l}\text { Cappellari (2017) } \\
\text { Lazarotto (2017) } \\
\text { Welter (2018) } \\
\end{array}$ \\
\hline & & CHMI5 & $\begin{array}{l}\text { Orientação para } \\
\text { atualização } \\
\text { constante }\end{array}$ & $\begin{array}{l}\text { A organização possui a } \\
\text { habilidade de criar novos } \\
\text { modelos de gestão e de } \\
\text { estrutura organizacional }\end{array}$ & $\begin{array}{l}\text { Lazarotto (2017) } \\
\text { Welter (2018) }\end{array}$ \\
\hline & & CHMI6 & $\begin{array}{l}\text { Orientação para o } \\
\text { mercado }\end{array}$ & $\begin{array}{l}\text { A organização possui } \\
\text { orientação para o } \\
\text { mercado }\end{array}$ & $\begin{array}{l}\text { Lazarotto (2017) } \\
\text { Rosa (2016) } \\
\text { Silva (2016) } \\
\text { Welter (2018) } \\
\end{array}$ \\
\hline & & CHMI7 & $\begin{array}{l}\text { Relacionamento } \\
\text { com clientes e } \\
\text { fornecedores }\end{array}$ & $\begin{array}{l}\text { A organização possui } \\
\text { um } \\
\text { relacionamento com os } \\
\text { clientes e fornecedores }\end{array}$ & $\begin{array}{l}\text { Cappellari (2017) } \\
\text { Rosa (2016) } \\
\text { Schmitz (2018) } \\
\end{array}$ \\
\hline & & CHMI8 & Criatividade & $\begin{array}{l}\text { A organização possui } \\
\text { criatividade para inovar } \\
\text { no mercado em que atua }\end{array}$ & $\begin{array}{l}\text { Lazarotto (2017) } \\
\text { Rosa (2016) }\end{array}$ \\
\hline \multirow{7}{*}{ PRBI } & \multirow{7}{*}{$\begin{array}{c}\text { PROCESSOS E } \\
\text { ROTINAS DE BUSCA } \\
\text { E INOVAÇÃO }\end{array}$} & PRBI1 & $\begin{array}{l}\text { Feedback do } \\
\text { mercado }\end{array}$ & $\begin{array}{l}\text { A organização possui } \\
\text { rotinas de recebimento } \\
\text { de feedbacks de clientes }\end{array}$ & $\begin{array}{l}\text { Lazarotto (2017) } \\
\text { Welter (2018) }\end{array}$ \\
\hline & & PRBI2 & Brainstorm & $\begin{array}{l}\text { A organização utiliza a } \\
\text { técnica de brainstorm } \\
\text { para novas ideias }\end{array}$ & $\begin{array}{l}\text { Rosa (2016) } \\
\text { Welter (2018) }\end{array}$ \\
\hline & & PRBI3 & $\begin{array}{c}\text { Sistemática de } \\
\text { reuniões frequentes }\end{array}$ & $\begin{array}{l}\text { A organização realiza } \\
\text { reuniões frequentes }\end{array}$ & $\begin{array}{l}\text { Lazarotto (2017) } \\
\text { Rosa (2016) }\end{array}$ \\
\hline & & PRBI4 & $\begin{array}{c}\text { Controle da } \\
\text { qualidade }\end{array}$ & $\begin{array}{l}\text { A organização possui } \\
\text { rotinas de controle da } \\
\text { qualidade }\end{array}$ & $\begin{array}{l}\text { Rosa (2016) } \\
\text { Welter (2018) }\end{array}$ \\
\hline & & PRBI5 & $\begin{array}{l}\text { Gestão de } \\
\text { processos }\end{array}$ & $\begin{array}{l}\text { A organização realiza } \\
\text { revisão e melhorias } \\
\text { contínuas dos processos } \\
\text { organizacionais }\end{array}$ & $\begin{array}{l}\text { Rosa (2016) } \\
\text { Cappellari (2017) } \\
\text { Lazarotto (2017) } \\
\text { Welter (2018) } \\
\end{array}$ \\
\hline & & PRBI6 & $\begin{array}{c}\text { Respostas rápidas } \\
\text { às mudanças do } \\
\text { mercado }\end{array}$ & $\begin{array}{lr}\text { A organização responde } \\
\text { rapidamente às } \\
\text { mudanças do mercado } \\
\text { em que atua }\end{array}$ & $\begin{array}{l}\text { Cappellari (2017) } \\
\text { Silva (2016) }\end{array}$ \\
\hline & & PRBI7 & $\begin{array}{c}\text { Incorporação de } \\
\text { novas tecnologias e } \\
\text { metodologias de } \\
\text { trabalho } \\
\end{array}$ & $\begin{array}{l}\text { A organização incorpora } \\
\text { novas tecnologias e } \\
\text { processos produtivos }\end{array}$ & $\begin{array}{l}\text { Cappellari (2017) } \\
\text { Rosa (2016) } \\
\text { Silva (2016) } \\
\text { Welter (2018) } \\
\end{array}$ \\
\hline MAGC & $\begin{array}{l}\text { MECANISMOS DE } \\
\text { APRENDIZAGEM E }\end{array}$ & MAGC1 & Benchmarking & $\begin{array}{l}\text { A organização busca a } \\
\text { aprendizagem por meio }\end{array}$ & $\begin{array}{l}\text { Cappellari (2017) } \\
\text { Rosa (2016) } \\
\text { Welter (2018) } \\
\end{array}$ \\
\hline
\end{tabular}




\begin{tabular}{|c|c|c|c|c|}
\hline \multirow[t]{6}{*}{$\begin{array}{c}\text { GOVERNANÇA DO } \\
\text { CONHECIMENTO }\end{array}$} & & \multicolumn{3}{|c|}{$\begin{array}{l}\text { de troca de informações } \\
\text { com outras organizações }\end{array}$} \\
\hline & MAGC2 & $\begin{array}{c}\text { Parcerias de apoio } \\
\text { tecnológico }\end{array}$ & $\begin{array}{l}\text { A organização apoia e } \\
\text { coopera com pesquisas } \\
\text { de centros externos, com } \\
\text { a abertura de estágios e } \\
\text { com pesquisas } \\
\text { acadêmicas }\end{array}$ & $\begin{array}{l}\text { Cappellari (2017) } \\
\text { Rosa (2016) } \\
\text { Silva (2016) }\end{array}$ \\
\hline & MAGC3 & $\begin{array}{l}\text { Treinamentos } \\
\text { continuados }\end{array}$ & $\begin{array}{l}\text { A organização realiza } \\
\text { treinamentos } \\
\text { continuados das equipes } \\
\text { de trabalho }\end{array}$ & $\begin{array}{l}\text { Lazzarotto } \\
\text { (2017) } \\
\text { Rosa (2016) } \\
\text { Schmitz (2018) } \\
\end{array}$ \\
\hline & MAGC4 & $\begin{array}{c}\text { Sistema de } \\
\text { informações } \\
\text { gerenciais }\end{array}$ & $\begin{array}{l}\text { A organização integra e } \\
\text { compartilha as } \\
\text { informações e os } \\
\text { conhecimentos internos } \\
\text { entre os funcionários e } \\
\text { gestores }\end{array}$ & $\begin{array}{l}\text { Cappellari (2017) } \\
\text { Lazarotto (2017) } \\
\text { Rosa (2016) } \\
\text { Schmitz (2018) }\end{array}$ \\
\hline & MAGC5 & Comitês ad hoc & $\begin{array}{l}\text { A organização possui } \\
\text { comissões formadas por } \\
\text { funcionários } \\
\text { diferentes } \\
\text { departamentos para } \\
\text { resolver problemas e } \\
\text { propor projetos }\end{array}$ & $\begin{array}{l}\text { Lazarotto (2017) } \\
\text { Rosa (2016) } \\
\text { Silva (2016) }\end{array}$ \\
\hline & MAGC6 & $\begin{array}{l}\text { Incentivo à } \\
\text { formação }\end{array}$ & $\begin{array}{l}\text { A organização incentiva } \\
\text { a formação acadêmica e } \\
\text { profissional dos } \\
\text { funcionários }\end{array}$ & $\begin{array}{l}\text { Lazarotto (2017) } \\
\text { Rosa (2016) } \\
\text { Schmitz (2018) } \\
\text { Silva (2016) }\end{array}$ \\
\hline
\end{tabular}

Fonte: Elaborado pelos autores, com base nos dados da pesquisa (2019).

\section{Método}

Quanto à abordagem, esta pesquisa se classifica como quantitativa, de natureza descritiva. Quanto aos procedimentos técnicos, classifica-se como uma pesquisa bibliográfica e uma e-survey (Minayo, 2013).

Conforme Kump et al. (2018), na construção de uma escala de CD, esta deve: i) capturar a CD ampla e geral para não medir exemplos de CD específicas de um setor ou contexto organizacional; ii) ser eficaz e genérica para medir ambientes e mercados de alta velocidade, continuando útil mesmo com mudanças do mercado; e iii) possibilitar o desenvolvimento e a análise da vantagem competitiva. Desse modo, considerando que diferentes setores apresentam distintas formas de desenvolver e alcançar resultados de $\mathrm{CD}$, escolheu-se, neste estudo, uma amostragem que garantisse o máximo de variação nos dados, em relação tanto ao seu porte quanto ao seu setor de atuação.

As organizações selecionadas para a pesquisa foram delimitadas pelos setores de comércio, serviços e indústrias, sendo incluídas empresas de pequeno, médio e grande porte, conforme a classificação do Serviço Brasileiro de Apoio às Micro e Pequenas Empresas (SEBRAE, 2013). A região delimitada para análise é pertencente ao Conselho Regional de Desenvolvimento (COREDE) Noroeste Colonial do Estado do Rio Grande do Sul e atua predominantemente nos setores dinâmicos de agropecuária e metalomecânica (Fundação de Economia e Estatística [FEE], 2019). 
Os entrevistados consistem em membros da alta administração dessas organizações, pois, de acordo com Helfat et al. (2007) e Helfat e Martin (2015), as capacidades dinâmicas são perceptíveis em nível gerencial e da alta administração. Embora se assuma a limitação imposta pela utilização de um único informante, tendo em vista que as organizações, em função de tempo e custos, tendem a não aceitar convites para colaborar com estudos quando são solicitadas respostas de múltiplos informantes (Hubner \& Power, 1985) e tendo em vista o objetivo e o contexto de aplicação deste estudo, tal escolha foi realizada a fim de evitar a possível perda de organizações e informações.

Para cálculo do tamanho da amostra, avaliou-se o poder estatístico do tamanho da amostra, definindo-a a priori, com o auxílio do software G*Power 3.1.9.2 (Faul, Erdfelder, Lang, \& Buchner, 2009), em 55 casos. Porém, como a sugestão é usar o dobro ou o triplo desse valor para ter um modelo mais consistente (Ringle, Silva, \& Bido, 2014), a amostra calculada foi de 110 casos. O método de coleta de dados utilizado foi o questionário (método $e$-survey), estruturado em uma escala do tipo Likert de 7 pontos (que varia de $1=$ discordo totalmente a 7 = concordo totalmente).

Ao todo foram enviados 867 e-mails para as organizações da região delimitada $(4,9 \%$ da população total), as quais também receberam o convite e a explicação dos objetivos da pesquisa via $e$ survey. O período de coleta de dados ocorreu entre 02 de setembro e 08 de novembro de 2019, resultando em 137 (15,8\%) questionários válidos respondidos. Dos questionários retornados, 50,36\% foram respondidos por diretores, $23,36 \%$ por gestores administrativos, $15,33 \%$ por administradores, e $10,95 \%$ por funcionários em posição de liderança estratégica. Em relação ao setor, 45,99\% das organizações que responderam são do setor industrial, 29,93\%, do setor de serviços, e 24,09\%, do setor de comércio. Dessas empresas, $37,96 \%$ são de grande porte, $32,85 \%$ são de médio porte, e 29,20\% são de pequeno porte.

A análise, interpretação e validação dos dados foram realizadas por meio dos softwares Microsoft Excel 2019 para tabulação dos dados e SmartPLS 3.2.8 (Ringle, Wende, \& Will, 2005) para as análises do modelo de mensuração e do modelo estrutural com a técnica de Modelagem de Equações Estruturais. Tendo em vista a abordagem escolhida para este estudo, ressalta-se que as característicaschaves do PLS-SEM, como a capacidade de lidar com amostras pequenas, a possibilidade de testar modelos complexos e o fato de não presumir a normalidade dos dados, são comumente encontradas nas investigações das Ciências Sociais (Hair, Hult, Ringle, \& Sarstedt, 2014).

Para a estimação dos modelos, utilizou-se o esquema de ponderação Path Weighting Scheme, que pondera a correlação ou regressão múltipla de modo que a dimensão possa ser prevista e possa ser uma boa preditora da dimensão subsequente, considerando a direcionalidade do modelo estrutural (Hair et al., 2014). Após, para testar a significância das relações das dimensões deste estudo, usou-se o módulo Bootstrapping (BT), com os seguintes parâmetros: no sign change, 137 casos e 5000 reamostras (Hair et al., 2014; Ringle et al., 2014).

Para a verificação da relevância preditiva dos modelos, empregou-se o módulo Blindfolding (BD), uma técnica de reutilização da amostra que omite todas as distâncias dos pontos de dados nos 
indicadores dos construtos endógenos e estima os parâmetros com os dados remanescentes (Hair et al., 2014). Para a utilização do Blindfolding no PLS, é necessário primeiramente estimar a distância de omissão (D), sendo recomendado que essa distância varie entre cinco e dez e que esse valor não seja múltiplo do tamanho da amostra (Hair et al., 2014). Como a amostra deste estudo foi de 137, esta pesquisa utilizou o valor 7, que já é o número padrão estimado no PLS-SEM.

Inicialmente, a análise realizada voltou-se a certificar a validade dos dados, examinando-os por meio do processo de preparação, que englobou as seguintes etapas: verificação de dados faltantes (missing values), codificação e transformação dos dados e entrada dos dados no software de execução das análises (Hair, Tatham, Anderson, \& Black, 2005). Além disso, apesar de o PLS-SEM não ter restrições quanto à distribuição das variáveis (normalidade e linearidade) (Gefen, Straub, \& Boudreau, 2000; Hair et al., 2014), procedeu-se a um teste de normalidade por intermédio da estatística Kolmogorov-Smirnov. Como o valor da significância obtido em todas as variáveis foi inferior a 0,05, rejeitou-se a suposição de normalidade (Marôco, 2014), situação que reforça a opção de uso do PLS.

\section{Resultados}

A apresentação dos resultados inicia-se com a validação do modelo de mensuração da escala proposta. Em seguida, expõe-se a validação do modelo estrutural.

\section{Validação do modelo de mensuração}

Hair et al. (2014) destacam que o modelo de mensuração (outer model) é avaliado em relação a sua validade convergente (Average Variance Extracted - AVE), confiabilidade (confiabilidade composta - CC - e alpha de Cronbach) e validade discriminante (critério de Fornell-Lacker e CrossLoadings) para modelos reflexivos. A validade convergente e a confiabilidade foram avaliadas com base na AVE, na CC e no alpha de Cronbach de cada dimensão da escala. A AVE mede a percentagem da variância total dos indicadores que é explicada pela dimensão, cujo valor deve ser >0,50 (Hair et al., 2014), o que significa que a variável latente explica mais da metade da variância dos seus indicadores (Tabachnick \& Fidell, 2001). Já a CC avalia o quanto os indicadores suportam as dimensões (Fornell \& Larcker, 1981), tendo como referência > 0,70. E o alpha de Cronbach mensura a confiabilidade da consistência interna de uma escala, ou seja, avalia a magnitude em que os itens de um instrumento estão correlacionados (Hair et al., 2014).

A Tabela 2, a seguir, apresenta os valores das cargas fatoriais de cada indicador e os valores da AVE, da CC e do alpha de Cronbach de cada dimensão da escala. 
Tabela 2 - Cargas fatoriais, validade convergente e confiabilidade

\begin{tabular}{|c|c|c|c|c|c|c|}
\hline & \multicolumn{3}{|c|}{ CARGAS FATORIAIS } & \multirow[t]{2}{*}{ AVE } & \multirow[t]{2}{*}{$\mathrm{CC}$} & \multirow[t]{2}{*}{ Alpha } \\
\hline & CHMI & PRBI & MAGC & & & \\
\hline CHMI1 & 0,7878 & & & \multirow{8}{*}{0,5974} & \multirow{8}{*}{0,9221} & \multirow{8}{*}{0,9034} \\
\hline CHMI2 & 0,6981 & & & & & \\
\hline CHMI3 & 0,7511 & & & & & \\
\hline CHMI4 & 0,7936 & & & & & \\
\hline CHMI5 & 0,7984 & & & & & \\
\hline CHMI6 & 0,7763 & & & & & \\
\hline CHMI7 & 0,7253 & & & & & \\
\hline CHMI8 & 0,8436 & & & & & \\
\hline PRBI1 & & 0,7555 & & \multirow{7}{*}{0,6070} & \multirow{7}{*}{0,9151} & \multirow{7}{*}{$0,891 \mathrm{c}$} \\
\hline PRBI2 & & 0,7150 & & & & \\
\hline PRBI3 & & 0,7781 & & & & \\
\hline PRBI4 & & 0,7626 & & & & \\
\hline PRBI5 & & 0,8635 & & & & \\
\hline PRBI6 & & 0,7683 & & & & \\
\hline PRBI7 & & 0,8025 & & & & \\
\hline MAGC1 & & & 0,7222 & \multirow{6}{*}{0,6049} & \multirow{6}{*}{0,9015} & \multirow{6}{*}{0,8684} \\
\hline MAGC2 & & & 0,7999 & & & \\
\hline MAGC3 & & & 0,8621 & & & \\
\hline MAGC4 & & & 0,7956 & & & \\
\hline MAGC5 & & & 0,7320 & & & \\
\hline MAGC6 & & & 0,7457 & & & \\
\hline
\end{tabular}

Fonte: Elaborado pelos autores, com base nos dados da pesquisa (2019).

As informações apresentadas mostram que todos os valores da AVE, da CC e do alpha de Cronbach são, respectivamente, $>0,50,>0,70$ e $>0,70$, o que permite afirmar que o modelo de mensuração da escala apresenta validade convergente e confiabilidade (Hair et al., 2014). Em continuidade, avalia-se a validade discriminante da escala.

Segundo Hair et al. (2014), a escala tem validade discriminante se cada um dos indicadores obtiver em suas próprias dimensões cargas superiores às de qualquer outra dimensão. Na Tabela 3, que apresenta as cargas cruzadas, é possível verificar que todos os indicadores das dimensões têm valores superiores relativos aos correspondentes Cross-Loadings, o que assegura a sua validade discriminante. Os resultados sugerem que as afirmativas utilizadas são, de fato, indicadores observáveis das próprias dimensões propostas na escala. 
Tabela 3 - Cross-Loadings dos indicadores da escala

\begin{tabular}{ll|cc}
\hline & \multicolumn{1}{c}{ CHMI } & PRBI & MAGC \\
\hline CHMI1 & $\mathbf{0 , 7 8 7 8}$ & 0,4797 & 0,3979 \\
CHMI2 & $\mathbf{0 , 6 9 8 1}$ & 0,5201 & 0,4786 \\
CHMI3 & $\mathbf{0 , 7 5 1 1}$ & 0,475 & 0,3058 \\
CHMI4 & $\mathbf{0 , 7 9 3 6}$ & 0,6982 & 0,5942 \\
CHMI5 & $\mathbf{0 , 7 9 8 4}$ & 0,6949 & 0,6399 \\
CHMI6 & $\mathbf{0 , 7 7 6 3}$ & 0,5937 & 0,4526 \\
CHMI7 & $\mathbf{0 , 7 2 5 3}$ & 0,4623 & 0,3616 \\
CHMI8 & $\mathbf{0 , 8 4 3 6}$ & 0,6484 & 0,5237 \\
PRBI1 & 0,3851 & $\mathbf{0 , 7 5 5 5}$ & 0,6201 \\
PRBI2 & 0,5104 & $\mathbf{0 , 7 1 5 0}$ & 0,4818 \\
PRBI3 & 0,4774 & $\mathbf{0 , 7 7 8 1}$ & 0,5975 \\
PRBI4 & 0,4679 & $\mathbf{0 , 7 6 2 6}$ & 0,5237 \\
PRBI5 & 0,7196 & $\mathbf{0 , 8 6 3 5}$ & 0,6666 \\
PRBI6 & 0,6706 & $\mathbf{0 , 7 6 8 3}$ & 0,5793 \\
PRBI7 & 0,7778 & $\mathbf{0 , 8 0 2 5}$ & 0,6589 \\
MAGC1 & 0,5894 & 0,5836 & $\mathbf{0 , 7 2 2 2}$ \\
MAGC2 & 0,3146 & 0,5007 & $\mathbf{0 , 7 9 9 9}$ \\
MAGC3 & 0,4299 & 0,6653 & $\mathbf{0 , 8 6 2 1}$ \\
MAGC4 & 0,6724 & 0,5809 & $\mathbf{0 , 7 9 5 6}$ \\
MAGC5 & 0,3253 & 0,6354 & $\mathbf{0 , 7 3 2 0}$ \\
MAGC6 & 0,5006 & 0,5718 & $\mathbf{0 , 7 4 5 7}$ \\
\hline
\end{tabular}

Nota 1: Aas cargas cruzadas de cada indicador estão sinalizadas em negrito.

Fonte: Elaborado pelos autores, com base nos dados da pesquisa (2019).

Na segunda etapa da validação discriminante, utilizou-se o critério de Fornell-Larcker ao comparar as raízes quadradas dos valores de AVEs de cada dimensão com as correlações das dimensões (Henseler, Ringle, \& Sinkovics, 2009). Nesse critério, os valores na diagonal principal (raiz quadrada da AVE) devem ser superiores aos valores nas respectivas linhas e colunas (correlações), evidenciando a presença de validade discriminante (Hair et al., 2014). A Tabela 4, a seguir, apresenta as correlações entre dimensões, na qual é possível verificar que a raiz quadrada da AVE de cada dimensão foi superior às correlações em todas as dimensões, indicando, assim, a validade discriminante entre elas.

Tabela 4 - Matriz de correlação e validade discriminante (critério de Fornell-Larcker)

\begin{tabular}{ccccc}
\hline & \multicolumn{2}{c}{ CHMI } & PRBI & MAGC \\
\hline CHMI & $\mathbf{0 , 7 7 2 9}$ & & \\
\cline { 2 - 3 } PRBI & 0,7511 & $\mathbf{0 , 7 7 9 1}$ & \\
MAGC & 0,6205 & 0,7611 & $\mathbf{0 , 7 7 7 8}$ \\
\hline
\end{tabular}

Nota 1: A raiz quadrada da AVE está distribuída ao longo da diagonal principal, em negrito.

Nota 2: Correlações iguais ou superiores a 0,126 são significantes a 5\%, e correlações iguais ou superiores a 0,166 são significantes a $1 \%$.

Fonte: Elaborado pelos autores, com base nos dados da pesquisa (2019). 
Uma vez que as etapas da validação do modelo de mensuração da escala foram verificadas e atendidas, prossegue-se para a validação do modelo estrutural, que é apresentada na próxima seção.

\section{Validação do modelo estrutural}

Segundo Hair et al. (2014), para a avaliação do modelo estrutural, quatro critérios devem ser analisados: 1) coeficientes de determinação $\left(\mathrm{R}^{2}\right)$ das dimensões; 2) estimativas para os coeficientes de caminho (sinal, magnitude e significância); 3) relevância preditiva $\left(\mathrm{Q}^{2}\right)$; e 4) tamanho dos efeitos de mediação $\left(\mathrm{f}^{2}\right)$. Assim, para avaliar o poder explicativo do modelo estrutural da escala, utiliza-se a variância explicada $\left(\mathrm{R}^{2}\right)$ de todas as suas dimensões. Para a área de Ciências Sociais e Comportamentais, Cohen (1977) sugere que $\mathrm{R}^{2}=2 \%$ seja classificado como efeito pequeno, $\mathrm{R}^{2}=13 \%$, como efeito médio e $\mathrm{R}^{2}=26 \%$, como efeito grande. Com os valores de $\mathrm{R}^{2}$ encontrados, é possível verificar que o $\mathrm{R}^{2} \mathrm{da}$ dimensão CHMI é 0,802 , o que significa que $80,2 \%$ de sua variação pode ser explicada pelo modelo estudado. O R ${ }^{2}$ da dimensão PRBI é 0,874 , de forma que $87,4 \%$ de sua variação pode ser explicada pelo modelo estudado. Já o $\mathrm{R}^{2}$ da dimensão MAGC é 0,744 , de modo que $74,4 \%$ de sua variação pode ser explicada pelo modelo estudado. Portanto, todas as dimensões analisadas apresentam valores de $\mathrm{R}^{2}$ com efeito grande (Cohen, 1997).

A seguir, a significância das relações é apresentada na Tabela 5, em que se verificam os valores do teste $t$-Student, cujos resultados devem ser superiores a 1,96 para que a escala seja adequada com nível de significância de $5 \%$.

Tabela 5 - Significância das relações estruturais

\begin{tabular}{cccc}
\hline Relação Estrutural & Coeficiente Estrutural & Erro-Padrão & t-Student \\
\hline CD -> CHMI & 0,8951 & 0,0208 & $43,1086^{*}$ \\
CD -> PRBI & 0,9359 & 0,0104 & $89,5894^{*}$ \\
CD -> MAGC & 0,8635 & 0,0275 & $31,3639^{*}$ \\
\hline
\end{tabular}

Nota 1: * = nível de significância 1\% (=>2,58).

Fonte: Elaborado pelos autores, com base nos dados da pesquisa (2019).

Assim, conforme os valores do $t$-Student, o nível de significância das dimensões da escala mostra-se superior a 1,96, demonstrando que o modelo é adequado e possui nível de significância de $5 \%$. Por fim, faz-se a análise dos indicadores de qualidade de ajuste dos modelos, ambos obtidos pelo uso do módulo Blindfolding: relevância ou validade preditiva $\left(\mathrm{Q}^{2}\right)$ ou indicador de Stone-Geisser e tamanho do efeito $\left(\mathrm{f}^{2}\right)$ ou indicador de Cohen.

$\mathrm{O}$ primeiro indicador de $\left(\mathrm{Q}^{2}\right)$ avalia a qualidade da predição do modelo, tendo como critério valores maiores que zero (Hair et al., 2014). Já o segundo indicador ( $\mathrm{f}^{2}$ ) avalia o quanto cada dimensão da escala é "útil" para o ajuste do modelo, com base nos seguintes valores de referência: 0,02 , que é considerado pequeno, 0,15 , que é considerado médio, e 0,35, que é considerado grande (Hair et al., 2014). A Tabela 6 apresenta os valores de $\mathrm{Q}^{2}$ e $\mathrm{f}^{2}$ para as dimensões da escala. 
Tabela 6 - Relevância preditiva e tamanho do efeito

\begin{tabular}{ccc}
\hline & $\mathbf{Q}^{2}$ & $\mathbf{f}^{2}$ \\
\hline CHMI & 0,4676 & 0,5604 \\
PRBI & 0,5213 & 0,5945 \\
MAGC & 0,4402 & 0,6133 \\
\hline
\end{tabular}

Fonte: Elaborado pelos autores, com base nos dados da pesquisa (2019).

Na simulação com uma distância de omissão de 7, verifica-se que no modelo analisado todos os valores de $\mathrm{Q}^{2}$ são $>0$, constatando-se, assim, que a escala apresenta validade preditiva. Igual situação foi verificada no indicador de qualidade de ajuste $\mathrm{f}^{2}$, em que todas as dimensões apresentam valor $>0,35$, o que indica que todos elas são importantes para o ajuste geral da escala proposta neste estudo.

\section{Conclusões}

$\mathrm{Na}$ busca por entender como as organizações desenvolvem CD para sustentar a vantagem competitiva, este estudo teve como objetivo propor e validar uma escala de mensuração do desenvolvimento de $\mathrm{CD}$, por meio de seus elementos componentes e mecanismos organizacionais. $\mathrm{A}$ escala final para mensuração do desenvolvimento dessas capacidades apresentou três dimensões e vinte indicadores.

O desenvolvimento e a validação da escala indicaram resultados consonantes com os previstos por Meirelles e Camargo (2014). Desse modo, as dimensões da escala analisada comportam indicadores que possibilitam analisar o desenvolvimento de comportamentos e habilidades de mudança e inovação, processos e rotinas de busca e inovação e mecanismos de aprendizagem e governança do conhecimento que dão suporte ao desenvolvimento de CD em organizações.

Entre as contribuições teóricas deste estudo, destaca-se a possibilidade de novas investigações empíricas sobre $\mathrm{CD}$, que poderão ser desenvolvidas ou ampliadas usando a escala proposta e validada neste estudo. Ressalta-se, também, a possibilidade de desenvolvimento da temática das CD em estudos voltados a relacionar o desenvolvimento de CD com outros constructos, como os de abordagem de equações estruturais, a partir da escala. Com isso, por meio da utilização da escala, novos insights fundamentados na teoria e empiria podem ser realizados.

De maneira prática, a contribuição da escala para fins gerenciais permite a identificação de pontos fracos, relacionados às dimensões e aos indicadores, de modo que os gestores podem direcionar seus esforços. Além disso, pode ser utilizada para possibilitar às organizações investirem em mecanismos organizacionais que lhes garantirão vantagem competitiva por intermédio do desenvolvimento de CD.

Por fim, é preciso citar algumas limitações deste estudo, como o fato de que a amostragem considerada para as análises integra uma região específica do Brasil, razão pela qual seus resultados 
devem ser interpretados e replicados com cautela. Outra limitação diz respeito à escolha dos setores selecionados para amostra, sendo necessários estudos adicionais para validar os achados em setores específicos. Há, ainda, a limitação da seleção de um único respondente por organização, mesmo que justificada neste estudo.

Assim, futuros estudos devem adaptar, estender ou descartar itens da escala tendo em vista a especificidade da região ou do setor a ser estudado. Com relação ao tamanho da amostra, apesar de esta pesquisa ter alcançado resultados significativos recomenda-se que estudos futuros utilizem amostras maiores. Sugere-se, ainda, a replicação e revalidação da escala em outros contextos, principalmente em setores com ambientes mais dinâmicos e competitivos, fator preditor do desenvolvimento de CD.

\section{Referências}

Ambrosini, V., \& Bowman, C. (2009). What are dynamic capabilities and are they a useful construct in strategic management? International Journal of Management Reviews, 11(1), $29-49$.

Andreeva, T., \& Chaika, V. (2006). Dynamic capabilities: what they need to be dynamic? St Petersburg, Russia: St. Petersburg State University.

Araújo, C. C. S., Pedron, C. D., \& Bitencourt, C. (2018). Identifying and assessing the scales of dynamic capabilities: a systematic review. Revista de Gestão, 25(4), 390-412.

Barney, J. B. (1991). Firm resources and sustained competitive advantage. Journal of Management, 17(1), 99-120.

Barreto, I. (2010). Dynamic capabilities: a review of past research and an agenda for the future. Journal of Management, 36(1), 256-280.

Cappellari, G. (2017). Inteligência de mercado e desenvolvimento de capacidades dinâmicas: relações na gestão estratégica dos negócios (Dissertação de mestrado). Universidade Regional do Noroeste do Rio Grande do Sul, Ijuí, Brasil.

Cohen, J. (1997). Statistical power analysis for the behavioral sciences (Rev. ed.). New York, NY: Academic Press.

Collis, D. J. (1994). Research note: how valuable are organizational capabilities? Strategic Management Journal, 15, 143-152.

Corrêa, R. O., Bueno, E. V., Karto, H. T., \& Silva, L. M. D. O. (2018). Dynamic managerial capabilities: scale development and validation. Managerial and decision economics, 40(1), 3-15.

D’Ávila, O. S., \& Silveira-Martins, O. (2017). Proposição/validação de escala de mensuração da capacidade dinâmica produtiva. Revista eletrônica de estratégia e negócios, 10(1), 285-311.

Danneels, E. (2010). Trying to become a different type of company; dynamic capability at Smith Corona. Strategic Management Journal, 32(1), 235-264.

Easterby-Smith, M., \& Prieto, I. M. (2008). Dynamic capabilities and knowledge management: an integrative role for learning? British Journal of Management, 19(3), 235-249.

Eisenhardt, K. M., \& Martin, J. A. (2000). Dynamic capabilities: what are they? Strategic Management Journal, 21(10-11), 1105-1121. 
Eriksson, T. (2014). Processes, antecedents and outcomes of dynamic capabilities. Scandinavian Journal of Management, 30(1), 65-82.

Faul, F., Erdfelder, E., Lang, A.-G., \& Buchner, A. (2007). G*Power 3: a flexible statistical power analysis program for the social, behavioral, and biomedical sciences. Behavior Research Methods, 39, 175-191.

Ferreira, M. A. S. P. V., Reis, N. R., Serra, F. A. R., \& Costa, B. K. (2014). Entendendo a pegada da VBR nos estudos em negócios internacionais: os últimos vinte anos de pesquisa. Brazilian Business Review, 11(4), 57-90.

Fornell, C., \& Larcker, D. F. (1981). Evaluating structural equation models with unobservable variables and measurement error. Journal of Marketing Research, 18(1), 39-50.

Fundação de Economia e Estatística (2019). Corede Noroeste Colonial. Recuperado de https://www.fee.rs.gov.br/perfil-socioeconomico/coredes/detalhe/?corede=Noroeste+Colonial

Gefen, D., Straub, D., \& Boudreau, M. (2000). Structural equation modeling and regression: guidelines for research practice. Communications of AIS, 4(7), 1-79.

Gerard, J. A. A. (2011). Theory of organizational routines: development of a topology and identification of contextual determinants. Ann Arbor, Michigan: ProQuest UMI Dissertation Publishing.

Guerra, R. M. A., Tondolo, V. A. G., \& Camargo, M. E. (2016). O que (ainda) podemos aprender sobre capacidades dinâmicas. Revista Ibero-Americana de Estratégia, 15(1), 44-64.

Hair, J. F., Anderson, R. E.; Tatham, R. L., \& Black, W. (2005). Análise multivariada de dados (5a ed.). Porto Alegre, RS: Bookman.

Hair, J. F., Hult, G. T. M., Ringle, C. M., \& Sarstedt, M. (2014). A primer on partial least squares Structural Equation Modeling (PLS-SEM). Thousand Oaks, California: Sage.

Helfat, C. E. (1997). Know-how and asset complementarity and dynamic capability accumulation: the case of R\&D. Strategic Management Journal, 18(5), 339-360.

Helfat, C. E., \& Martin, J. A. (2015). Dynamic managerial capabilities: Review and assessment of managerial impact on strategic change. Journal of Management, 41(5), 1281-1312.

Helfat, C., Finkelstein, S., Mitchell, W., Peteraf, M., Singh, H., Teece, D., \& Winter, S. (2007). Dynamic capabilities: understanding strategic changes in organizations. Malden, Massachusetts: Blackwell Publishing.

Henseler, J., Ringle, C., \& Sinkovics, R. (2009). The use of partial least squares path modeling in international marketing. Advances in International Marketing (AIM), 20, 277-320.

Huber, G. P., \& D. J. Power. (1985). Retrospective reports of strategic managers: guidelines for increasing their accuracy. Strategic Management Journal, 6, 171-180.

Kump, B., Engelmann, A., Kessler, A., \& Schweiger, C. (2018). Toward a dynamic capabilities scale: measuring organizational sensing, seizing, and transforming capacities. Industrial and Corporate Change, 28(5), 1149-1172.

Kurtmollaiev, S. (2020). Dynamic capabilities and where to find them. Journal of Management Inquiry, 29(1), 3-16. 
Lazarotto, E. C. (2017). Processo de desenvolvimento de capacidades dinâmicas em organizações do setor hospitalar (Dissertação de mestrado). Universidade de Passo Fundo, Passo Fundo, Brasil.

Marcus, A. A., \& Anderson, M. H. (2006). A general dynamic capability: does it propagate business and social competencies in the retail. Journal of Management Studies, 43(1), 19-46.

Marôco, J. (2014). Análise estatística com o SPSS Statistics. Lisboa, Portugal: ReportNumber.

Martins, S. E., \& Tavares, P. M. (2014). Processo de formulação de estratégias: capacidade mercadológica, incerteza ambiental e desempenho. Organizações em Contexto, 10(20), 297-322.

Meirelles, D. S., \& Camargo, A. A. B. (2014). Capacidades dinâmicas: o que são e como identificálas? Revista de Administração Contemporânea, 18 [Edição Especial], 41-64.

Minayo, M. C. S. (2013). Pesquisa social: teoria, método e criatividade (33a ed.). Petrópolis, RJ: Vozes.

Penrose, E. T. (1959). The theory of the growth of the firm (3a ed.). Oxford, UK: Oxford University Press.

Raman, A., \& Bharadwaj, S. S. (2017). Dynamic service capabilities enabling agile services: Scale development and initial validation in Indian services industry. Jornal of Eterprise Information Management, 30(1), 166-187.

Ringle, C. M., Silva, D., \& Bido, D. (2014). Modelagem de equações estruturais com utilização do SmartPLS. Revista Brasileira de Marketing, 13(2), 56-73.

Ringle, C. M., Wende, S., \& Will, A. (2005). SmartPLS 3.2.8. Hamburg: SmartPLS. Recuperado de http://www.smartpls.com

Rosa, K. C. (2016). O Desenvolvimento de capacidades dinâmicas como recurso estratégico em uma organização do segmento do vestuário (Dissertação de mestrado), Faculdade Meridional, Passo Fundo, Brasil.

Sausen, J. O. (2012). Gestão estratégica, competitividade e desenvolvimento. In D. B. Sieden (Org.), Desenvolvimento sob múltiplos olhares (pp. 207-267). Ijuí, RS: Unijuí.

Schilke, O., Hu, S., \& Helfat, C. E. (2018). Quo vadis, dynamic capabilities? A content-analytic review of the current state of knowledge and recommendations for future research. Academy of Management Annals, 12(1), 390-439.

Schmitz, R. (2018). Mecanismos e instrumentos de desenvolvimento de capacidades adaptativas em Instituições de Ensino Superior privadas da região Noroeste do Estado do Rio Grande do Sul (Dissertação de mestrado), Faculdade Meridional, Passo Fundo, Brasil.

Serviço Brasileiro de Apoio às Micro e Pequenas Empresas (2013). Anuário do trabalho na micro e pequena empresa 2013. Recuperado de http://www.sebrae.com.br/Sebrae/Portal\%20Sebrae/Anexos/Anuario\%20do\%20Trabalho\%20Na\%2 0Micro\%20e\%20Pequena\%20Empresa_2013.pdf

Sher, P. J., \& Lee, V. C. (2004). Information technology as a facilitator for enchancing dynamic capabilities through knowledge management. Information and Management, 41(8), 933-945. 
Silva, R. L. (2016). Análise das capacidades dinâmicas em uma empresa do setor de confecção têxtil: um estudo de caso da empresa MiraSul (Dissertação de mestrado), Faculdade Meridional, Passo Fundo, Brasil.

Silveira, B. R., \& Silveira-Martins, E. (2017). Capacidade dinâmica do agronegócio: proposta e validação de escala para mensuração. In 20 Seminários em Administração, São Paulo, Brasil: SEMEAD.

Silveira-Martins, E., \& Zonatto, V. C. S. (2015). Proposição e validação de escala para mensuração da capacidade turística. Turismo em análise, 26(4), 745-773.

Tabachnick, B., \& Fidell, L. (2001). Using multivariate statitstics (4a ed.). Boston, Massachusetts: Allyn and Bacon.

Teece, D. (2007). Explicating dynamic capabilities: the nature and microfoundations of (sustainable) enterprise performance. Strategic Management Journal, 28(13), 1319-1350.

Teece, D. (2009). Dynamic capabilities \& strategic management. Oxford, UK: Oxford University Press.

Teece, D. J., \& Pisano, G. (1994). The dynamic capabilities of firms: an introduction. Industrial and Corporate Change, 3(3), 537-556.

Teece, D. J., Pisano, G., \& Shuen, A. (1990). Firm capabilities, resources, and the concept of strategy. Berkeley, California: University of California.

Teece, D. J., Pisano, G., \& Shuen, A. (1997). Dynamic capabilities and strategic management. Strategic Management Journal, 18(7), 509-533.

Tondolo, V. A. G., Tondolo, R. R. P., Guerra, R. M. A., \& Camargo, M. E. (2018). Capacidades dinâmicas em organizações sem fins lucrativos: uma proposta de mensuração para o terceiro setor. Revista Eletrônica Científica do CRA-PR, 5(1), 18-33.

Vasconcelos, F. C., \& Cyrino, Á. B. (2000). Vantagem competitiva: os modelos teóricos atuais e a convergência entre estratégia e teoria organizacional. Revista de Administração de Empresas, 40(4), 20-37.

Wang, C. L., \& Ahmed. P. K. (2007). Dynamic capabilities: A review and research. International Journal of Management Reviews, 9, 31-51.

Welter, C. V. N. (2018). O desenvolvimento da capacidade inovativa como recurso estratégico em organizações egressas de incubadoras de base tecnológica (Dissertação de mestrado), Universidade Regional do Noroeste do Rio Grande do Sul, Ijuí, Brasil.

Winter, S. G. (1964). Economic 'natural selection' and the theory of the firm. Yale Economic Essays, 4, 225-272.

Winter, S. G. (2003). Understanding dynamic capabilities. Strategic Management Journal, 33(2), 91 101.

Zahra, S. A., \& George, G. (2002). Absorptive capacity: a review, reconceptualization, and extension. Academy of Management Review, 27(2), 185-203.

Zahra, S. A., Sapienza, H. J., \& Davidsson, P. (2006). Entrepreneurship and dynamic capabilities: a review, model and research agenda. Journal of Management Studies, 43(4), 917-955. 
Zaluski, F. C., Sausen, J. O., \& Ferreira, G. C. (2020). Proposição de um instrumento de mensuração do desenvolvimento de capacidades dinâmicas

Zollo, M., \& Winter, S. G. (2002). Deliberate learning and the evolution of dynamic capabilities.

Organization Science, 13(3), 339-351. 